\title{
The AnteOwl WR Intravascular Ultrasound-Guided Parallel Wiring Technique for Chronic Total Occlusion of Below-The-Knee Arteries
}

Naoki Hayakawa ( $\square$ haya.naoki1981@gmail.com )

Asahi General Hospital https://orcid.org/0000-0002-4168-2808

Satoshi Kodera

University of Tokyo Hospital: Tokyo Daigaku Igakubu Fuzoku Byoin

Masataka Arakawa

Asahi General Hospital

Satoshi Hirano

Asahi General Hospital

Yasunori Inoguchi

Asahi General Hospital

Junji Kanda

Asahi General Hospital

\section{Research Article}

Keywords: Chronic total occlusion, Endovascular therapy, Intravascular ultrasound, Below-the knee

Posted Date: February 23rd, 2022

DOI: https://doi.org/10.21203/rs.3.rs-1341109/v1

License: (9) (i) This work is licensed under a Creative Commons Attribution 4.0 International License.

Read Full License

Version of Record: A version of this preprint was published at CVIR Endovascular on March 26th, 2022.

See the published version at https://doi.org/10.1186/s42155-022-00294-2. 


\section{Abstract}

Background: Although endovascular therapy is used to treat chronic limb-threatening ischemia, long chronic total occlusion (CTO) is still challenging to treat. Especially in patients with poor run-off belowthe-knee (BTK) arteries, it is difficult to perform a retrograde approach, and even guidewire passage may be difficult.

Case presentation: We treated two cases of chronic limb-threatening ischemia using our novel extreme antegrade guidewire crossing technique by AnteOWI WR intravascular ultrasound (IVUS)-guided parallel wiring to a BTK artery (EXCAVATOR technique). Case 1 was a 70-year-old man with ulceration of the right toe. The AnteOwI WR IVUS was intentionally advanced into the subintimal space of the posterior tibial artery, and the totally intraplaque route was advanced by IVUS-guided parallel wiring that was successfully passed from the lateral plantar aspect to the true lumen of the digital artery. Case 2 was a 76-year-old woman with rest pain and cyanosis of the right lower limb. Angiography showed total occlusion from the superficial femoral artery to BTK arteries. AnteOwI WR IVUS-guided parallel wiring was repeatedly performed until the distal true lumen of the peroneal artery was reached, and revascularization was successfully achieved via the antegrade approach alone.

Conclusions: With its excellent crossable performance, good image quality, and high navigational ability within the CTO, the AnteOwI WR can be used to pass parallel wiring into the distal true lumen for BTK CTO.

\section{Background}

Endovascular therapy (EVT) is now a widespread method of revascularization for patients with chronic limb-threatening ischemia (CLTI); however, it is often difficult to use EVT to treat long chronic total occlusion (CTO) in below-the-knee (BTK) lesions (lida et al., 2017; Lyden et al., 2009). Although the success of EVT in such lesions has been improved by the introduction of retrograde approaches such as distal puncture, retrograde approaches are extremely difficult to use for lesions with poor distal target vessels (Schmidt et al., 2017). Furthermore, the presence of poor run-off vessels often makes it difficult to perform bypass surgery.

AnteOwI WR (AnteOwl) (TERUMO, Tokyo, Japan) is a new intravascular ultrasound (IVUS) specifically developed for CTO intervention (Figure 1A, B) (Okamura et al., 2020). This IVUS makes it easy to project the directionality of the target plaque onto angiography by utilizing the asymmetric structure of the IVUS transducer and guidewire (Figure 1C, D).

Herein, we report the application of our IVUS-guided parallel wiring technique for BTK lesions and devised a new technique in which the guidewire is passed through the distal true lumen by repeatedly using this technique to advance into the true lumen. We describe this novel technique of extreme antegrade guidewire crossing by the AnteOwI IVUS-guided parallel wiring to a BTK artery (EXCAVATOR technique). 


\section{Case Presentations}

\section{Case 1}

A 70-year-old man presented with rest pain, cyanosis, intractable ulceration, and in the right toe. The skin perfusion pressure was $21 \mathrm{mmHg}$ dorsally and $10 \mathrm{mmHg}$ at the plantar aspect. A 6-Fr guiding sheath (Destination ${ }^{\circledR}$ guiding sheath; TERUMO) was inserted into the right common femoral artery (CFA) via the ipsilateral antegrade approach. Control angiography showed total occlusion of the anterior tibial artery to the dorsalis pedis artery (DPA), and total occlusion of the posterior tibial artery (PTA) to the lateral plantar artery (PLA) (Figure 2A, 2B). A 0.014-inch guidewire (Gladius MGES ${ }^{\circledR}$ guidewire; Asahi Intec, Aichi, Japan) and a microcatheter (Caravel ${ }^{\circledR}$ microcatheter; Asahi Intec) seemed to be advanced into the subintimal space in the PTA CTO (Figure 2C). We advanced the AnteOwl into the CTO. IVUS showed that the guidewire was in the subintimal space from the middle part of the PTA CTO. We performed the IVUSguided parallel wiring technique using the AnteOwl. We converted the direction of IVUS findings to angiography using the rotational angiography and asymmetrical structure of the IVUS transducer. We advanced a 0.014-inch guidewire (Halberd $\AA$ guidewire; Asahi Intec) and microcatheter (Caravel ${ }^{\circledR}$ microcatheter; Asahi Intec) a few millimeters to the left (right anterior oblique side) of the IVUS on angiography, and advanced through the intraplaque route (Figure $2 \mathrm{D}-\mathrm{H})$. Thus, we finally succeeded in passing the guidewire into the distal true lumen of the digital artery, and used IVUS to confirm that the true lumen was secured (Figure 2l, 2J). Based on the external elastic membrane-based vessel diameter accurately measured by IVUS, the PLA was dilated with a $2.5 \times 200-\mathrm{mm}$ balloon (SHIDEN HP®; Kaneka, Tokyo, Japan), the distal and middle PTA was dilated with a $3.0 \times 200-\mathrm{mm}$ balloon (SHIDEN HP®), and the proximal PTA was dilated with a 4.0×100-mm balloon (SHIDEN HP®) (Figure 2K, 2L). Final angiography showed sufficient expansion of the target lesion and good antegrade flow and blood flow in the side branches (Figure 2M, 2N). Immediately after EVT, the cyanotic color of the skin on the right toe markedly improved. The wound on the right toe healed within 5 months. No additional EVT was required.

\section{Case 2}

A 76-year-old woman with diabetes mellitus presented with rest pain and cyanosis of both lower limbs. Her ankle-brachial index could not be measured in either leg. Contrast-enhanced computed tomography showed total occlusion of the right SFA to the popliteal artery (PopA) to the BTK arteries (Figure 3A, 3B).

We inserted a 6-Fr guiding sheath (Crossroads ${ }^{\circledR}$ guiding sheath; NIPRO, Tokyo, Japan) from the left common femoral artery. Control angiography showed moderate stenosis of the distal EIA and total occlusion of the SFA from just proximal to the BTK arteries (Figure 3C-E). The distal DPA was barely imaged, while the main trunk of the tibial and peroneal arteries was not imaged at all. We advanced a 0.014-inch guidewire (Jupiter X; Boston Scientific) and microcatheter (Ichibanyari PAD2® microcatheter; Kaneka) to the CTO lesion in the SFA. When the AnteOwl IVUS was inserted at the proximal PopA, it was 
in the subintimal space from the mid-SFA, so IVUS-guided parallel wiring was started using a 0.014-inch guidewire (Astato XS9-40® guidewire; Asahi Intec) with a microcatheter (Ichibanyari PAD2® microcatheter; Kaneka) (Figure 3F, 3G). We advanced the guidewire along the intraplaque route and reached the distal PopA. However, the total occlusion continued to the BTK arteries. We again performed IVUS-guided parallel wiring using a 0.014-inch guidewire (Halberd® guidewire; Asahi Intec) with a microcatheter (Ichibanyari PAD2® microcatheter; Kaneka) (Figure 3H, 3I). By repeating these steps of IVUS-guided parallel wiring several times, we finally succeeded in passing the guidewire through the distal true lumen of the peroneal artery (Figure 3J-L). Tip injection confirmed that the distal true lumen of the peroneal artery was connected to the DPA via a collateral tract (Figure 3M). According to the IVUS evaluation, a 10.0×80-mm stent (Epic®; Boston Scientific) was deployed for the EIA, a $7.0 \times 120-\mathrm{mm}$ drugeluting stent, $7.0 \times 120-\mathrm{mm}$ stent, $6.0 \times 120-\mathrm{mm}$ stent, and $6.0 \times 40-\mathrm{mm}$ stent (Eluvia $\AA$; Boston Scientific) were deployed for the SFA just proximal to the middle of the PopA, and a 4.0×150-mm drug-coated balloon (Ranger $\AA$; Boston Scientific) was dilated in the distal PopA. The peroneal artery was dilated with a 3.0×200-mm balloon (SHIDEN HP®; Kaneka). Final angiography showed very good antegrade blood flow to the periphery (Figure $3 \mathrm{~N}-\mathrm{R}$ ). The postoperative ankle-brachial index improved to 0.9 , and the clinical course was good.

\section{Discussion}

We reported successful revascularization for long CTO lesions with very poor run-off target vessels using the EXCAVATOR technique, which brings the IVUS as far distal as possible to the BTK CTO and uses parallel wiring to allow antegrade guidewire passage to the distal true lumen under IVUS guidance. We previously reported the feasibility of EVT for FP CTO using the AnteOwl IVUS-guided approach and described the method used to perform AnteOwl IVUS-guided intraplaque wiring in FP CTO (Hayakawa et al., 2021); however, to the best of our knowledge, this is the first report of the use of AnteOwl IVUS in BTK СTO intervention.

The primary strategy for BTK CTO is generally to use antegrade wiring; however, a previous clinical studies reported a relatively high rate of antegrade guidewire crossing failure (Kokkinidis et al., 2020; Tan et al., 2021). Various retrograde approaches have improved the success rate of the procedure in BTK CTO; however, lesions with poor distal target vessels are extremely difficult to treat with retrograde approaches (Schmidt et al., 2017). Recently, percutaneous deep venous arterialization (pDVA) was reported as a useful approach for patients with CLTI when conventional revascularization is not indicated. However, pDVA makes it difficult to preserve the tip of the toe and is not currently available in all centers (Schmidt et al., 2020).

To overcome these issues associated with treating complex BTK CTO lesions, we devised a novel antegrade AnteOwl IVUS-guided wiring technique called the EXCAVATOR. AnteOwl is a novel IVUS with an asymmetrical structure of the proximal transducer and IVUS wire, facilitating the reflection of IVUS findings onto angiographic images to allow accurate navigation of the second guidewire into the 
intraplaque route. AnteOwl has a small profile and crossable catheter, durable hydrophilic coating, and high-resolution imaging.

The steps of IVUS-guided parallel wiring are as follows. The guidewire is first advanced as far distally as possible in an antegrade fashion. Next, we advance the microcatheter as far distally as possible and use the bougie effect by microcatheter to insert the AnteOwl (Figure 4A). When the first guidewire is in the subintimal space, we manipulate the second guidewire into the intraplaque route under IVUS guidance (Figure 4B). The area that can be observed by IVUS should be confirmed by IVUS while proceeding. When the guidewire is advanced to the area that cannot be observed by IVUS, we step down to a floppy or intermediate type of guidewire and advance this guidewire under angiographic guidance. By repeating this process and proceeding along the intraplaque route, the guidewire can finally be passed through the distal lumen (Figure 4C, 4D).

In recent years, IVUS has been used to determine the appropriate vessel diameter for BTK lesions, and clinically, it has accelerated improvements in wound care (Fujihara et al., 2020; Soga et al., 2021). In addition to the evaluation of the appropriate vessel diameter, the AnteOwl may also allow guidewire passage in difficult cases and reduce the incidence of distal puncture.

This AnteOwl-guided guidewire crossing has limitations because severe calcification may not enable the passage of the IVUS or may be poorly observed by IVUS. A much larger study is required to confirm the efficacy of AnteOwl IVUS-guided EVT for BTK CTO.

\section{Conclusion}

AnteOwI IVUS was effective for antegrade guidewire crossing to treat BTK CTO. The EXCAVATOR technique can be used to treat long BTK CTO with poor target vessels.

\section{Abbreviations}

CTO, chronic total occlusion; BTK, below-the-knee; IVUS, intravascular ultrasound; EXCAVATOR, extreme antegrade guidewire crossing by AnteOWI WR intravascular ultrasound-guided parallel wiring to a BTK artery; PTA, posterior tibial artery; SFA, superficial femoral artery; EVT, endovascular therapy; CLTI, chronic limb-threatening ischemia; FP, femoropopliteal; PDVA, percutaneous deep venous arterialization; EIA, external iliac artery; DPA, dorsalis pedis artery; PLA, plantar artery; PopA, popliteal artery; CFA, common femoral artery

\section{Declarations}

Ethics approval and consent to participate: All procedures were performed in accordance with the ethical standards of the institutional and/or national research committees and the 1964 Declaration of Helsinki and its later amendments or comparable ethical standards. 
Consent for publication: Written informed consent was obtained from the patients for publication of this case report and any accompanying images.

Availability of data and materials: The datasets used and/or analyzed during the current study are available from the corresponding author on reasonable request.

Competing interests: The authors declare that they have no competing interests.

Funding: No specific grants from any funding agency in the public, commercial, or not-for-profit sectors were received for this study.

Authors' contributions: $\mathrm{NH}, \mathrm{MA}, \mathrm{SH}$, and $\mathrm{YI}$ performed the procedures and pre- and post-procedure followups. SK drafted the manuscript and revised it critically for important intellectual content. JK provided final approval of the submitted manuscript. All authors read and approved the final manuscript.

\section{Acknowledgments}

We thank Kelly Zammit, BVSc, from Edanz Group (https://jp.edanz.com/ac), for editing a draft of this manuscript.

\section{References}

1. lida O, Takahara M, Soga Y, et al (2017) Three-year outcomes of surgical versus endovascular revascularization for critical limb ischemia: the SPINACH study (surgical reconstruction versus peripheral intervention in patients with critical limb ischemia). Circ Cardiovasc Interv 10(12): e005531. doi:10.1161/CIRCINTERVENTIONS.117.005531.

2. Lyden SP (2009) Techniques and outcomes for endovascular treatment in the tibial arteries. J Vasc Surg 50(5):1219-23. doi: 10.1016/j.jvs.2009.02.019.

3. Schmidt A, Bakker OJ, Bausback Y, et al (2017) The tibiopedal retrograde vascular access for challenging popliteal and below-the-knee chronic total occlusions: literature review and description of the technique. J Cardiovasc Surg (Torino) 58:371-82.

4. Okamura A, Iwakura K, Iwamoto $M$, et al (2020) Tip detection method using the new IVUS facilitates the 3-dimensional wiring technique for CTO intervention. Cardiovasc Interv 13(1):74-82.

5. Hayakawa N, Kodera S, Takanashi K, et al (2021) Efficacy of navigating through the intraplaque route using AnteOWI WR intravascular ultrasound in femoropopliteal chronic total occlusion. CVIR Endovasc 4(1):1-7. doi: 10.1186/s42155-021-00228-4.

6. Kokkinidis DG, Strobel A, Jawaid O, et al (2020) Development and validation of a predictive score for anterograde crossing of infrapopliteal chronic total occlusions: (the infrapop-CTO score). Catheter Cardiovasc Interv 95(4):748-55.

7. Tan M, Ueshima D, Urasawa K, et al (2021) Prediction of successful guidewire crossing of below-theknee chronic total occlusions using a Japanese scoring system. J Vasc Surg 74(2):506-13. 
8. Schmidt A, Schreve MA, Huizing E, et al (2020) Midterm outcomes of percutaneous deep venous arterialization with a dedicated system for patients with no-option chronic limb-threatening ischemia: the ALPS multicenter study. J Endovasc Ther 27(4):658-65. doi 10.1177/1526602820922179.

9. Fujihara M, Yazu Y, Takahara M (2020) Intravascular ultrasound-guided interventions for below-theknee disease in patients with chronic limb-threatening ischemia. J Endovasc Ther 27(4):565-74.

10. Soga Y, Takahara M, Ito N, et al (2021) Clinical impact of intravascular ultrasound-guided balloon angioplasty in patients with chronic limb threatening ischemia for isolated infrapopliteal lesion. Catheter Cardiovasc Interv 97(3):E376-84.

\section{Figures}

A

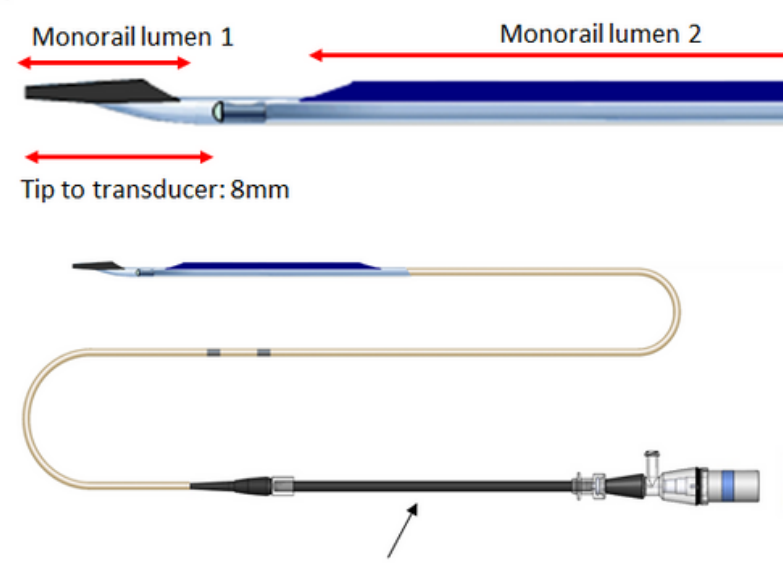

Auto pull back system
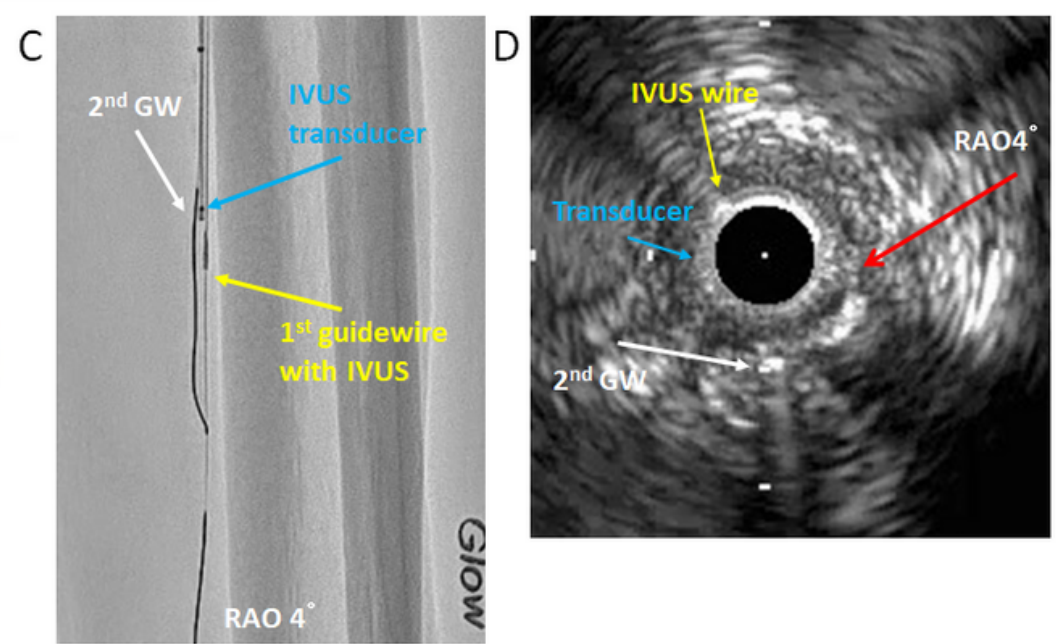

B

\begin{tabular}{|c|c|c|c|c|c|c|c|}
\hline & $\begin{array}{c}\text { Outer diameter of } \\
\text { window position } \\
\text { (mm) }\end{array}$ & $\begin{array}{l}\text { Shaft outer } \\
\text { diameter } \\
(\mathrm{mm})\end{array}$ & $\begin{array}{l}\text { Sensor position } \\
(\mathrm{mm})\end{array}$ & $\begin{array}{l}\text { Effective length } \\
\text { (cm) }\end{array}$ & $\begin{array}{l}\text { Frequency } \\
\text { (MHz) }\end{array}$ & $\begin{array}{l}\text { Pull back speed } \\
\text { (mm/s) }\end{array}$ & $\begin{array}{l}\text { Rotation speed } \\
\quad(\mathrm{rpm})\end{array}$ \\
\hline AnteOwl WR & $\begin{array}{c}0.86 \mathrm{~mm} \\
2.6 \mathrm{Fr} .\end{array}$ & $\begin{array}{l}1.03 \mathrm{~mm} \\
3.1 \mathrm{Fr} .\end{array}$ & $8 \mathrm{~mm}$ & $135 \mathrm{~cm}$ & 40 & $\begin{array}{l}0.5-9.0 \\
\text { (可変) }\end{array}$ & $1,800-5,400$ \\
\hline
\end{tabular}

\section{Figure 1}

A Structure of the AnteOwI WR (AnteOwI) intravascular ultrasound (IVUS). B Specifications of the AnteOwl. C Angiographic images of the AnteOwl in a below-the-knee lesion in the right anterior oblique (RAO) $4^{\circ}$ view. The $1^{\text {st }}$ guidewire (yellow arrow) and AnteOwl IVUS transducer (blue arrow) are in the posterior tibial artery. The $2^{\text {nd }}$ guidewire (GW) is indicated by the white arrow on the left side of the IVUS (right anterior oblique side). D IVUS findings of the chronic total occlusion lesion. The detector direction where the transducer and target plaque are maximally separated is at RAO4 (red arrow). Blue arrow: IVUS transducer; yellow arrow: IVUS wire ( $1^{\text {st }}$ guidewire); white arrow: $2^{\text {nd }} \mathrm{GW}$ successfully advanced into the target plaque. 


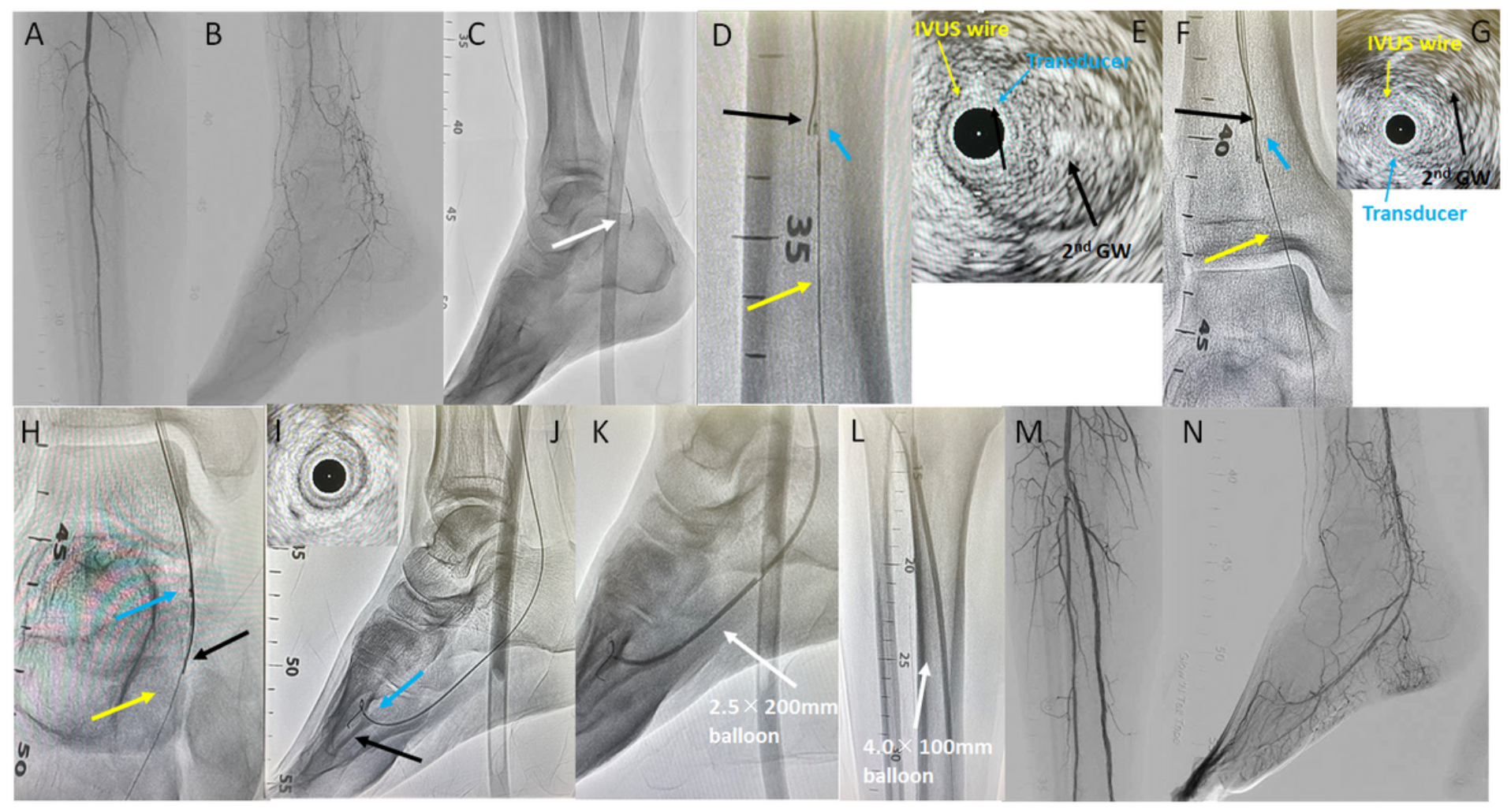

Figure 2

A, B Control angiography shows total occlusion of both tibial arteries, and very poor inframaleolar arteries. C A 0.014-inch guidewire (GW) is advanced into the subintimal space (white arrow: GW tip) in the posterior tibial artery (PTA). D, F, H Intravascular ultrasound (IVUS)-guided parallel wiring (D: proximal-tomiddle PTA; F: distal PTA; H: lateral plantar artery). Yellow arrow: $1^{\text {st }} \mathrm{GW}$ and IVUS catheter; blue arrow: IVUS transducer; black arrow: $2^{\text {nd }} \mathrm{GW}$ advanced into the intraplaque of the PTA. E, G IVUS findings of the chronic total occlusion (CTO) lesion in the PTA (E: proximal-to-middle PTA; G: distal PTA). Yellow arrow: IVUS wire; blue arrow: IVUS transducer; black arrow: $2^{\text {nd }} \mathrm{GW}$ in the intraplaque route. I IVUS findings in the distal part of the lateral plantar artery. The IVUS transducer is almost in the center of the vessel. $\mathbf{J}$ The $2^{\text {nd }}$ GW (black arrow) is advanced into the distal true lumen of the digital artery. Blue arrow: IVUS transducer. K A 2.5 $\times 200-\mathrm{mm}$ balloon is dilated in the lateral plantar artery to the distal PTA. L A 4.0×100-mm balloon is dilated in the proximal PTA. M, N Final angiography shows sufficient dilation, antegrade blood flow, and small branches. 


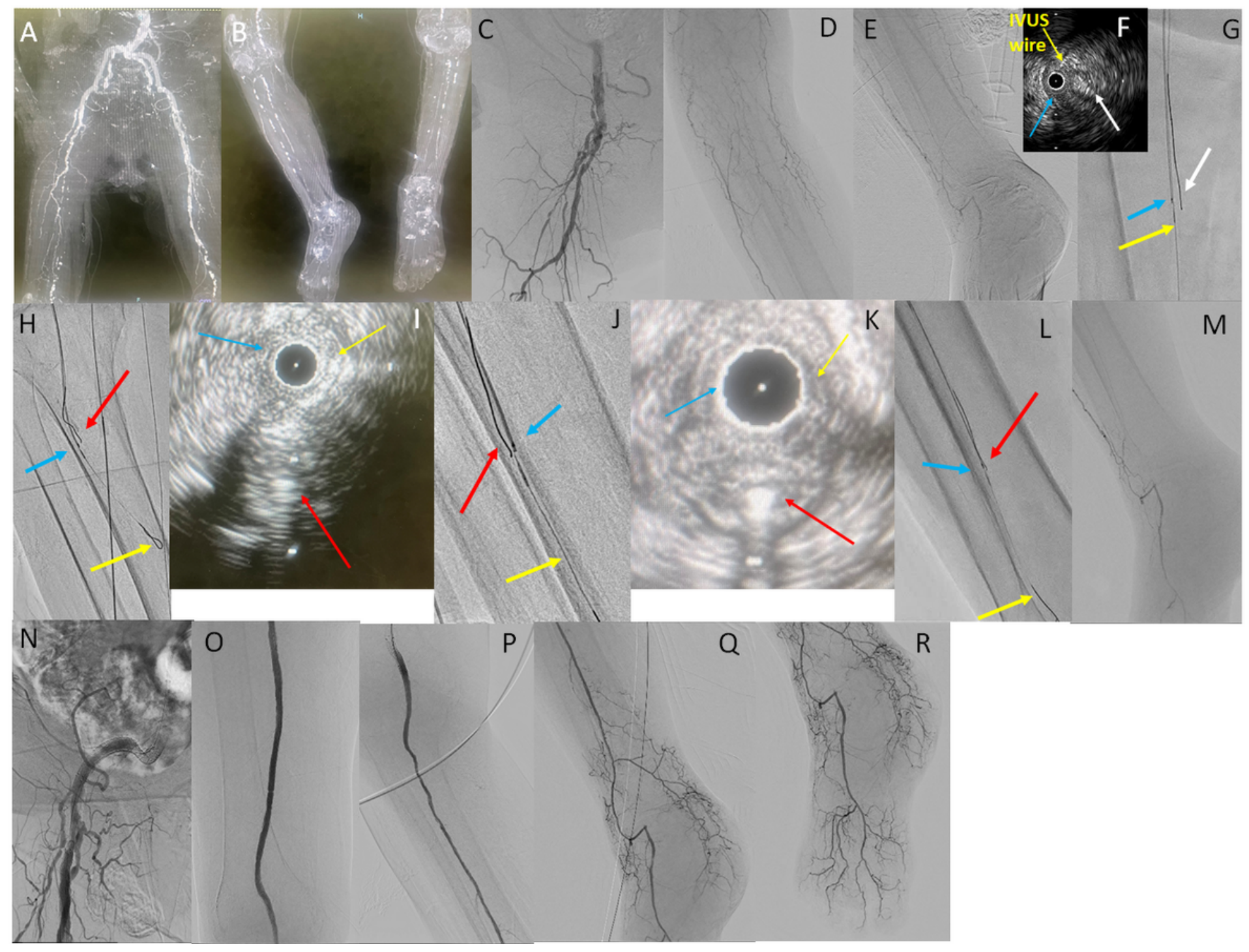

Figure 3

A, B Preprocedural enhanced computed tomography. C, D, E Control angiography shows total occlusion from the right superficial femoral artery (SFA) to below-the-knee arteries. Distal target vessels are very faint. F IVUS findings of the CTO lesion in the SFA. Yellow arrow: IVUS wire; blue arrow: IVUS transducer; white arrow: $2^{\text {nd }} \mathrm{GW}$ in the intraplaque route. $\mathbf{G}$ Intravascular ultrasound (IVUS)-guided parallel wiring (G: SFA). Yellow arrow: $1^{\text {st }}$ guidewire (GW) and IVUS catheter; blue arrow: IVUS transducer; white arrow: $2^{\text {nd }}$ GW advanced into the intraplaque of the chronic total occlusion (CTO) lesion. H, J, L IVUS-guided parallel wiring ( $\mathbf{H}$ : distal popliteal artery to peroneal artery; $\mathbf{J}$ : peroneal artery; $\mathbf{L}$ : distal peroneal artery). Yellow arrow: $1^{\text {st }} \mathrm{GW}$; blue arrow: IVUS transducer; red arrow: $2^{\text {nd }} \mathrm{GW}$ advanced into the intraplaque of the CTO lesion. I, K IVUS findings of the CTO lesion in the below-the-knee artery (I: distal popliteal artery to peroneal artery; K: peroneal artery). Yellow arrow: IVUS wire; blue arrow: IVUS transducer; red arrow: $2^{\text {nd }}$ GW in the intraplaque. M Tip injection from the distal peroneal artery shows the distal true lumen of below-the-ankle arteries. N, O, P, Q, R Final angiography shows sufficient antegrade blood flow. 
A

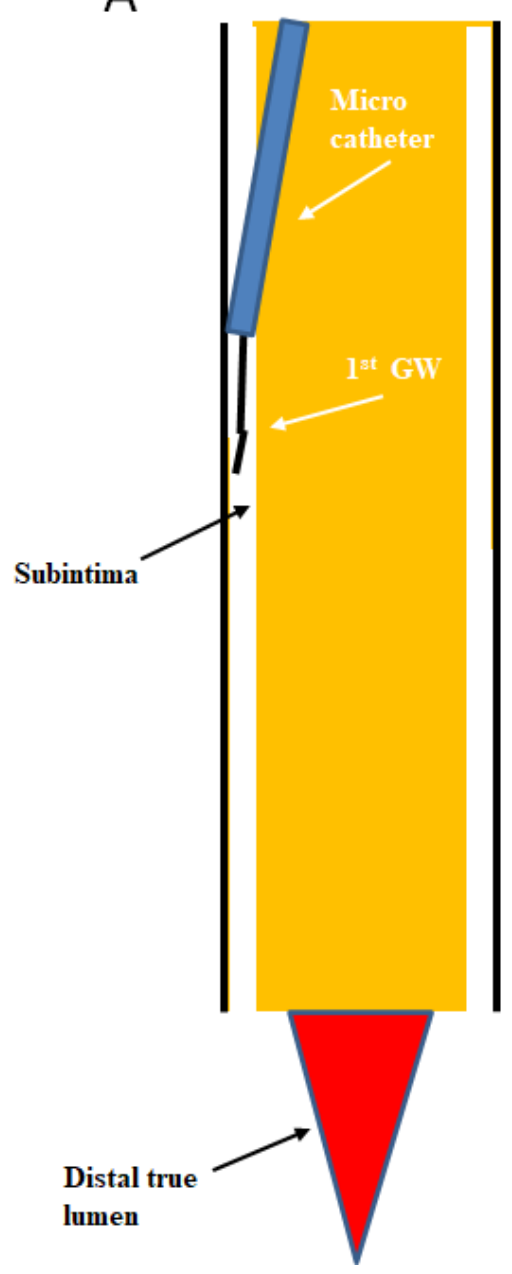

B

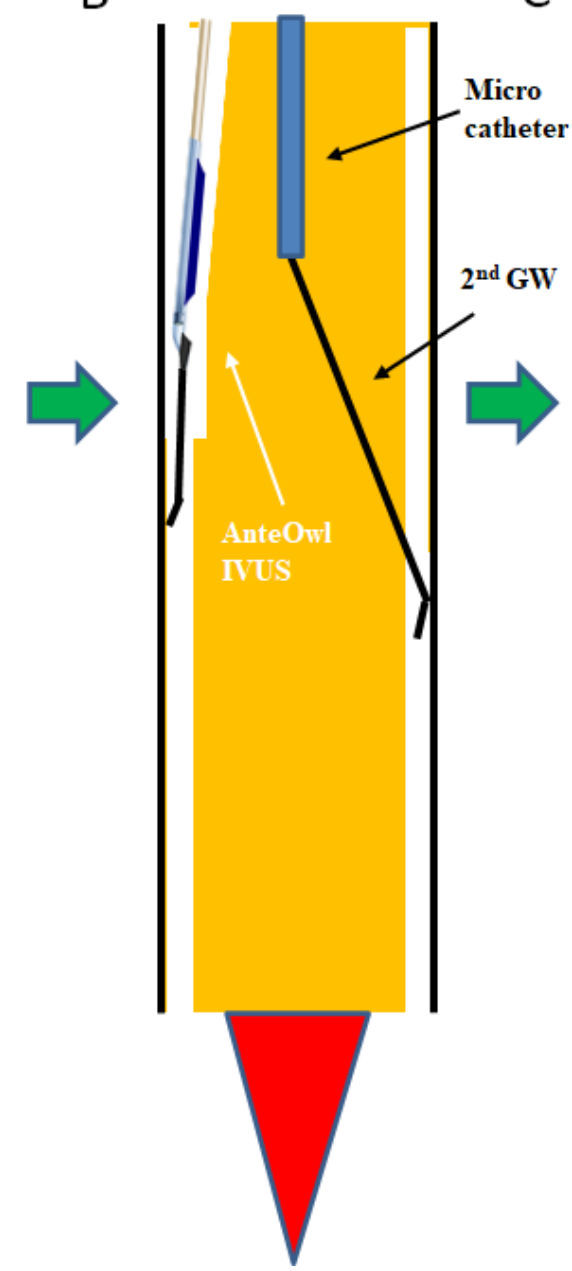

C

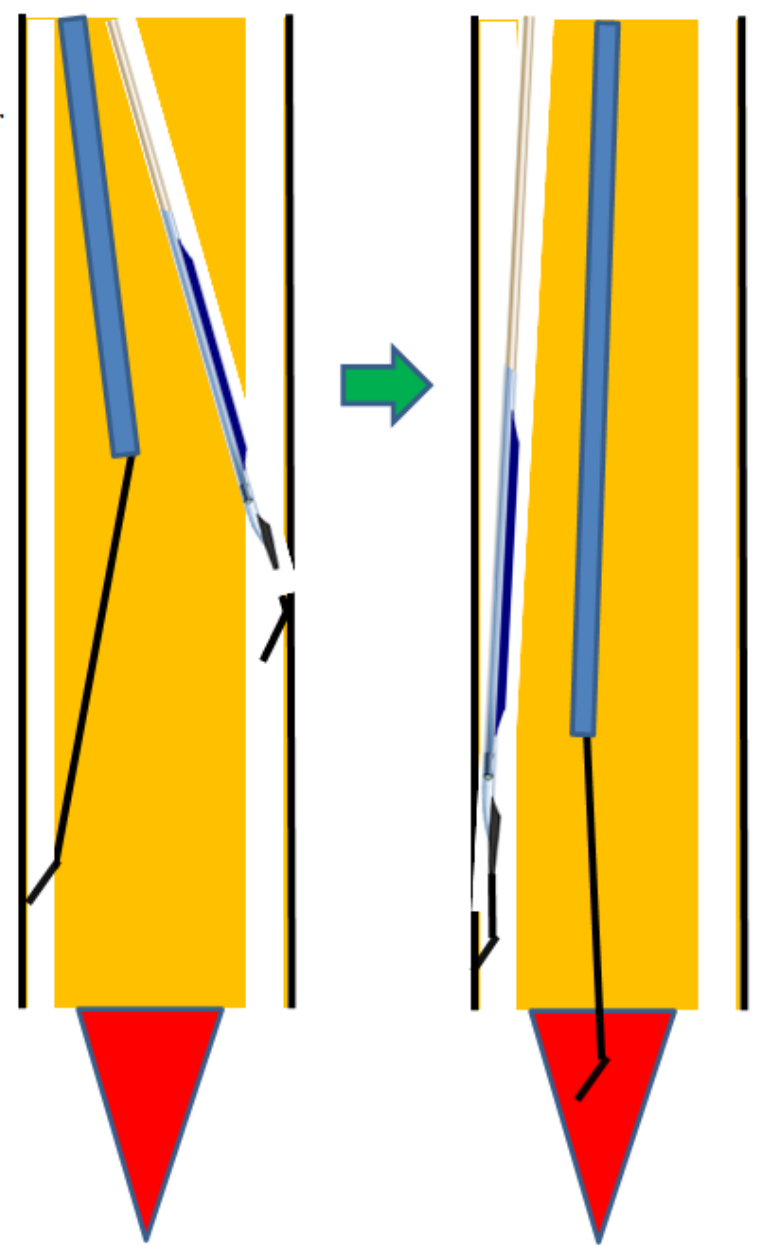

Figure 4

Schematic diagram of extreme antegrade guidewire crossing by AnteOwI WR intravascular ultrasound (IVUS)-guided parallel wiring to a below-the-knee artery (EXCAVATOR). A The $1^{\text {st }}$ guidewire (GW) with microcatheter is advanced into the subintima. We use the bougie technique with the microcatheter to prepare for IVUS insertion. B The AnteOwl IVUS is advanced along the $1^{\text {st }} \mathrm{GW}$. The $2^{\text {nd }} \mathrm{GW}$ with microcatheter is inserted to perform IVUS-guided parallel wiring. The $2^{\text {nd }} \mathrm{GW}$ is guided to the intraplaque under real-time IVUS guidance. The $2^{\text {nd }} \mathrm{GW}$ is then advanced into the subintima. $\mathbf{C}$ The bougie technique is used as shown in A and the IVUS is returned to the subintima. Under IVUS guidance, the $2^{\text {nd }} \mathrm{GW}$ is again introduced into the plaque. D Finally, the $2^{\text {nd }} \mathrm{GW}$ is passed from the distal plaque to the distal true lumen. 